\title{
ESCRIBIR TEXTOS ARGUMENTATIVOS
}

\author{
Aurora Martínez Ezquerro \\ Dra. en Filología Hispánica
}

\begin{abstract}
RESUMEN. La argumentación forma parte de nuestra vida cotidiana y se manifiesta en las expresiones lingüísticas desde la más temprana edad; es más, su aprendizaje supera los simples objetivos de una enseñanza disciplinaria y tiene importantes dimensiones filosóficas, psicológicas, sociales y culturales. Teniendo en cuenta estos aspectos, resulta muy interesante y, por supuesto, útil explotar sus diferentes posibilidades en el aula.

En el presente artículo se ofrece el desarrollo de la unidad didáctica "Escribir textos argumentativos" realizada con alumnos de 4. curso de Educación Secundaria Obligatoria. La experiencia tiene como objetivos fundamentales -teniendo en cuenta que nos encontramos en una etapa de afianzamiento- profundizar en las técnicas y procedimientos argumentativos, así como realizar una conceptualización de la argumentación y de sus estructuras. Las bases psicopedagógicas del trabajo se fundamentan en el aprendizaje constructivo y significativo; es más, el profesor se convierte en orientador, colaborador y planificador.
\end{abstract}

PALABRAS CLAVE: lengua castellana, enseñanza, argumentación, aprendizaje constructivo.

ABSTRACT. Argumentation is part of our daily lives. It manifests itself in our linguistic utterances from a very tender age. Learning to argue goes beyond the simple goals of a disciplinary education and has important philosophical, psychological, social and cultural dimensions. Taking all these aspects into account, classroom analysis of the different possibilities of argumentation turns out to be very interesting and extremely useful.

The present article is based on the development of a didactic unit, "Writing argumentative texts", which was carried out among children studying fourth year of ESO (Spanish Secondary Obligatory Education). Bearing in mind that this is a moment in which children's self confidence is strengthened, this experiment has two basic objectives: firstly, to make a deep analysis of argumentative techniques and procedures; secondly, to try and conceptualize the process of argumentation and its structures. This study is grounded on psycho-pedagogical bases which consider the learning process to be constructive and significant and reduce the role of the teacher to advising, collaborating, and plannifying activities to be developed by children in class.

KEYWORDS: Spanish language, teaching, argumentation, constructive learning.

\section{Introducción}

Una de las funciones básicas de la asignatura de Lengua Española ha sido tradicionalmente la enseñanza de la lengua escrita, si bien en pocas ocasiones se había 
considerado como un objeto de estudio en sí misma. Las posibilidades de cambio que nos propone el nuevo sistema educativo -recordemos la noción de currículum flexible y abierto- permiten atisbar una interesante y fructífera posibilidad de trabajar esta área en nuestras aulas.

La unidad de trabajo que desarrollo corresponde a la argumentación. ¿Por qué? Por muchas razones. La argumentación forma parte de la vida cotidiana y se manifiesta en las expresiones lingüísticas desde la más temprana edad, pues es inseparable el proceso de socialización de su desarrollo intelectual. Los niños desde pequeños tratan de convencer a sus padres para conseguir algo, justifican, en cierta medida, sus deseos. Por otro lado, no hay que olvidar que la enseñanza de la argumentación supera los simples objetivos de una enseñanza disciplinaria y tiene unas dimensiones filosóficas, psicológicas, sociales y culturales. La argumentación juega un papel importante en la educación ética del ciudadano moderno ${ }^{1}$, pues vive inmerso en una situación constante de persuasión.

Es tradicional en la enseñanza la ausencia de la argumentación en los diseños curriculares. Mi interés por el tema pretende, pues, llenar esta laguna "temática" que se observa con bastante generalidad. Concretamente, me he centrado en la enseñanza de la argumentación en Educación Secundaria, si bien en esta etapa se preende consolidar lo aprendido en las anteriores.

Para situarnos en el nivel correspondiente, es necesario exponer las diferentes etapas en las que se debe organizar el trabajo de la argumentación en el aula; corresponden éstas a las siguientes fases:

1. Etapa de iniciación al trabajo con la argumentación: comprende los cursos de

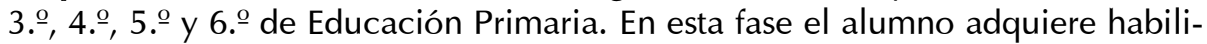
dades que necesitará para la posterior producción de textos argumentativos.

2. Etapa de desarrollo en el aprendizaje de la argumentación: comprende el primer ciclo de Educación Secundaria Obligatoria. Ahora se desarrolla fundamentalmente la teoría del texto.

3. Etapa de consolidación de los conocimientos y habilidades adquiridos sobre la argumentación: comprende el segundo ciclo de Educación Secundaria Obligatoria y el Bachillerato. Se trabaja directamente sobre textos argumentativos orales y escritos, se utilizan las técnicas adecuadas para su planteamiento y planificación, se emplean los distintos tipos de argumentos y se crean, asimismo, distintos tipos de textos argumentativos.

Pues bien, en esta tercera etapa es en la que voy a encuadrar la unidad didáctica

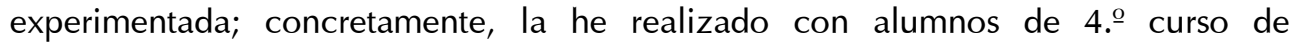
Educación Secundaria Obligatoria, y los objetivos que hemos perseguido -y alcanzado- son los siguientes:

- Analizar y definir previamente la situación de argumentación.

- Planificar los medios para una argumentación.

1. CAMPS, A. y DOLZ, J. (1995): “Introducción. Enseñar a argumentar: un desafío para la escuela actual". Comunicación, Lenguaje y Educación, 25, pág. 5. 
- Formular claramente la tesis que se pretende defender.

- Apoyar los argumentos con datos objetivos.

- Diferenciar argumentos de tópicos.

- Emplear distintas técnicas persuasivas.

- Practicar la contraargumentación empleando técnicas de concesión y refutación.

- Elaborar estructuradamente distintos tipos de textos argumentativos.

- Elaborar mensajes publicitarios para distintos tipos de comunicación.

- Analizar lingüísticamente distintos textos argumentativos.

En Educación Secundaria Obligatoria se profundiza en las técnicas y procedimientos argumentativos y se realiza un conceptualización de la argumentación y sus estructuras; mientras que en Bachillerato se profundiza en el análisis de los elementos argumentativos presentes en el discurso científico, periodístico y publicitario.

\section{Desarrollo de la unidad didáctica "Escribir textos argumentativos"}

\subsection{Fases}

La unidad didáctica que he aplicado se divide en cuatro fases, que son las siguientes:

1. Fase de actuación: se inspira en los enfoques basados en las funciones y en el proceso.

2. Fase de reflexión: toma como referencia el enfoque basado en el contenido.

3. Fase de análisis: se ha tenido en cuenta el enfoque que parte de la gramática.

4. Fase de generalización: se inspira en los mismos enfoques que la fase de actuación.

Esta unidad se desarrolló a lo largo de ocho sesiones, con un grupo de 4.․ curso de Educación Secundaria Obligatoria del I.E.S. "Esteban Manuel de Villegas" de Nájera (La Rioja). Aunque la mayoría de las sesiones se realizaron en la clase habitual, ocasionalmente, el desarrollo de las actividades exigió cambiar ese espacio ${ }^{2}$ y trasladarse a la biblioteca y a las aulas de audiovisuales y de informática.

\subsection{Objetivos}

El objetivo y punto de partida de esta unidad se origina en las necesidades concretas del alumnado, que a menudo suele manifestar en sus escritos errores de índole diversa: abundantes faltas de ortografía, escasos elementos de unión entre las oraciones, frecuentes confusiones en el uso de la puntuación, párrafos incoherentes, falta de creatividad, pobreza de léxico...

2. La infraestructura del centro es, términos generales, aceptable: aula de tecnología, aula de música, aula de audiovisuales, aula de plástica, aula de informática, aula de Proyecto Atenea, tres laboratorios (Física, Química y Ciencias Naturales), mini-laboratorio de foto-vídeo, sala de usos múltiples, gimnasio, 11 despachos de Departamento, sala de visitas, Biblioteca, sala de profesores y tres almacenes de material. 
Con esta unidad se pretende abordar estas cuestiones de una forma global e integradora, centrada en los procesos de redacción (planificación, textualización y revisión) aprovechando la virtualidad lógica del discurso argumentativo, con el fin de explotar la relación entre pensamiento y sintaxis, atendiendo a las relaciones entre proposiciones y lógica del discurso. También me propuse utilizar los medios de comunicación, el vídeo y el casete, como instrumentos de análisis de las producciones orales referidas al texto argumentativo.

\subsection{La unidad didáctica en el marco de los proyectos de centro}

\subsubsection{Proyecto Educativo de Centro}

Como ya se ha señalado, esta unidad está destinada a alumnos de 15 años de cuarto curso de E.S.O. Estos adolescentes se encuentran en el tramo de la enseñanza obligatoria y, aparte de la difícil etapa evolutiva en la que se hallan, ofrecen una diversidad que es preciso afrontar en el aula (interés, motivación...). Es más, el área de Lengua Castellana y Literatura es común y troncal. Estas peculiaridades hacen que el modelo de enseñanza-aprendizaje de la materia deba ser activo y motivador, debe relacionar lo ya aprendido con lo que van a aprender y, a su vez, debe intentar que lo tratado sea útil tanto a los que no van a seguir estudiando como a los que continúen en los Bachilleratos. Además esta área es integradora y necesariamente multidisciplinar, por eso quizá como en ninguna otra deben mantenerse los criterios de currículum abierto, flexible y realista.

Para ayudar en esta difícil labor educativa, en la evaluación inicial de principio de curso, en los ERPAS de los alumnos y durante el proceso educativo llevado a cabo en los meses anteriores a la puesta en marcha de la unidad, el profesor detecta las diferencias y las necesidades especiales de los alumnos, aspectos que se tienen en cuenta para la aplicación de la presente unidad.

El Centro se ubica en Nájera3 ; este I.E.S. cuenta en la actualidad con 44 profesores, 560 alumnos y 21 grupos. El entorno social al que pertenecen estos alumnos corresponde a la clase media rural. Los alumnos proceden en su mayoría de Nájera, pero también de pequeños núcleos rurales próximos a la localidad. Los padres son agricultores, empleados, obreros especializados, pequeños empresarios o profesionales.

El nivel cultural de los padres no es homogéneo y, por tanto, tampoco el de los hijos. También es diverso el nivel de intereses, aunque en su mayoría se detecta en los cursos altos una aspiración a terminar estudios medios con una nota numérica que les permita entrar en las Facultades que elijan. También hay que decir que esa aspiración -que les podría llevar a dedicar muchas horas de estudio- no se traduce en un amor al estudio por su valor formativo.

3. Localidad situada al suroeste de Logroño, a 24 kms., cuenta con una población aproximada de 6.800 habitantes y una superficie de 39'87 kms.2. Es capital histórica de La Rioja y ciudad de origen prerromano. Constituye un centro agrícola y es un importante mercado comarcal, origen de una industrialización modesta (muebles, cerámicas, cartonajes y productos alimenticios). 


\subsubsection{Proyecto Curricular de Centro}

La propuesta inicial que ofrece el MEC en el Diseño Curricular Base presenta un carácter flexible, abierto y orientador, de forma que permite al profesorado adoptar una gran parte de decisiones particulares en sus programaciones.

La realidad ha sido que cuando esa filosofía y psicopedagogía del Diseño Curricular Base se ha plasmado en leyes y normas, el MEC ha ido girando, quizá obligado por el hecho de que debía asegurar determinados contenidos mínimos para todos los adolescentes del Estado, y casi cerrando ese diseño base, hasta llegar a su máximo punto en la Resolución de 5 de marzo de 1992 por la que se regula la elaboración de proyectos curriculares para la Educación Secundaria Obligatoria. Según la resolución citada, los centros elaborarán proyectos curriculares para la ESO, de acuerdo con el currículo oficial establecido, e incluirá una serie de elementos que el artículo segundo especifica.

De acuerdo con esto, el Proyecto Curricular de Centro, dentro del que se integra la presente unidad didáctica, tiene los siguientes caracteres:

1. Se parte del conocimiento del currículo prescriptivo que garantiza unos niveles mínimos de igualdad.

2. La concepción de las áreas y de las materias se apoya en tres tipos de contenidos: conceptos, procedimientos y actitudes.

3. Se tienen en cuenta las características esenciales del entorno sociocultural y familiar en el que se desenvuelven los alumnos.

4. En la propuesta de currículo se favorece el respeto y la adaptación a las peculiaridades de los alumnos, a sus motivaciones, intereses y capacidades de aprendizaje. Se consideran los procesos de desarrollo psicológico que caracteriza al grupo de alumnos de referencia.

5. El currículo propuesto permite una secuenciación de contenidos en espiral, para trabajar contenidos idénticos o semejantes en distintos niveles de profundización.

6. Las unidades didácticas respetan, en términos generales, los ejes temáticos de sus áreas.

7. Las unidades didácticas establecen conexiones entre los contenidos de los distintos bloques.

8. La extensión de las unidades es variable, pero básicamente se ciñe a dos tipos: unas de larga duración (8 horas) y otras de menos duración (3 ó 4 horas).

9. Se presta especial atención a los conocimientos previos de los alumnos. Por eso las unidades tendrán sus contenidos secuenciados e interconexionados con los cursos, ciclos y etapas anteriores y posteriores.

2.4. Relación de la unidad didáctica con otras unidades didácticas de Lengua Castellana y Literatura y conexiones con los contenidos de las etapas, ciclos y cursos anteriores y posteriores

Esta unidad didáctica engloba otros bloques de contenido, tanto en la Educación Secundaria Obligatoria como en el Bachillerato, tales como la expresión oral, la gramática, el léxico, el análisis literario y los medios audiovisuales de comunicación. 


\subsubsection{Relación con los contenidos de la Educación Secundaria Obligatoria \\ BLOQUE 2: "Usos y formas de la comunicación escrita" \\ Conceptos:}

2. Estructuras textuales argumentativas. Aquí se pueden incluir los textos que presentan la estructura secuencial argumentativa: carta publicitaria, carta de opinión, carta de solicitud, artículo crítico, editorial secuencias textuales producidas en diálogos, entrevistas, coloquios, debates, conversaciones, que expresan una toma de decisiones, una postura ante algo, la defensa de una idea...

Procedimientos:

1. Comprensión de textos escritos de distinto tipo.

2. Análisis de textos escritos de distinto tipo.

3. Comentario escrito de textos escritos de distinto tipo.

4. Preparación, realización y evaluación de textos escritos de distinto tipo.

6. Utilización de textos escritos con distintas finalidades.

\section{Actitudes:}

1. Valoración de la lengua como instrumento para satisfacer una amplia gama de necesidades de comunicación.

2. Tratamiento de la información. Conecta con el primer ciclo: Planificación de los trabajos y búsqueda de información.

8. Actitud crítica ante temas de discriminación social, racial, sexual... Conecta con el primer ciclo: Participación, cooperación y solidaridad.

BLOQUE 3: "La lengua como objeto de conocimiento"

Conceptos:

3. Mecanismos de la lengua en el discurso, lo mismo se trabaja en el primer ciclo pero, lógicamente, con menor profundidad.

\section{Procedimientos:}

2. Utilización de marcas de adecuación del texto al contenido y de procedimientos de cohesión textual.

\section{Actitudes:}

1. Valoración de la lengua como producto y proceso sociocultural que evoluciona como vehículo de transmisión y creación cultural.

BLOQUE 4: "La literatura"

Conceptos:

3. Los géneros literarios: El ensayo y otros géneros literarios. 
Procedimientos:

4. Elaboración de un juicio personal argumentado sobre algunos textos literarios.

Actitudes:

7. Valoración crítica ante las determinaciones sociales que condicionan el consumo de textos literarios.

BLOQUE 5: "Sistemas de comunicación verbal y no verbal"

Conceptos:

4. Los medios de comunicación.

Procedimientos:

3. Manipulación de diferentes tipos de lenguajes no verbales.

Actitudes:

6. Actitud crítica ante los usos discursivos.

\subsubsection{Relación con los contenidos del Bachillerato}

\section{Contenidos:}

1. La variedad de los dicursos: La comunicación escrita. Tipología de los textos escritos.

2. Los discursos en los procesos de aprendizaje: Comprensión y producción de textos científicos, culturales, técnicos, etc. Técnicas auxiliares para la comprensión y producción de textos usuales en la vida académica.

5. La reflexión sobre la lengua: El texto como unidad de sentido y forma de intercambio: adecuación, coherencia y cohesión.

\subsubsection{Relación con otras áreas y materias}

Argumentar consiste en provocar o en aumentar la adhesión de los oyentes o lectores a lo que se defiende, denominado tesis. Se argumenta en casi todas las disciplinas del saber, en el discurso político o forense y, por supuesto, en las exposiciones de clase y en multitud de trabajos escolares. Entiendo, pues, que esta unidad didáctica puede tener rendimiento en diferentes áreas o materias considerando que uno de los objetivos educativos generales de cualquier nivel consiste en elaborar criterios personales y razonados sobre diferentes cuestiones, esto es, argumentar de forma razonada sobre cuestiones que se estudian. Es un procedimiento de carácter general, si bien predomina en el ámbito de las Humanidades y Ciencias Sociales. En la ESO entronca con el área de Ciencias Sociales, Geografía e Historia de la ESO; y en el Bachillerato, con las materias de Historia, Historia del Arte, Historia del Mundo Contemporáneo, Filosofía y Psicología. 


\subsection{Objetivos de la unidad didáctica}

\subsubsection{Objetivos generales del área}

- Atender a la claridad expresiva y al orden lógico en la disposición, utilizando un léxico adecuado.

- Construir oraciones bien estructuradas.

- Aplicar las convenciones básicas de puntuación a los escritos.

- Respetar las normas de concordancia.

- Considerar la buena utilización de las reglas ortográficas.

- Entregar puntualmente los trabajos con una correcta presentación.

A través de estos objetivos he creado un pauta de evaluación de la secuencia con un cuadro de especificaciones que fue entregado a los alumnos después de la primera fase (ver documento "Pauta de evaluación de la unidad" en el apartado 2.9.1.

\subsubsection{Objetivos didácticos}

a) Objetivo básico (nivel mínimo):

- Componer un texto argumentativo estructurado, comprensible y convincente a partir de un tema determinado.

b) Objetivos relativos a la organización del texto:

- Establecer esquemas previos y planificar lo que se quiere expresar en el texto, seleccionando y organizando la información de acuerdo con la intencionallidad.

- Elegir y dar coherencia al enunciador, teniendo en cuenta al destinatario del texto.

- Escribir un borrador, corrigiéndolo cuantas veces sea necesario.

- Reconocer los mecanismos de cohesión textual.

- Identificar las relaciones lógicas entre oraciones, proposiciones y párrafos.

- Entregar una versión definitiva.

c) Objetivos respecto al contenido del texto:

- Aprehender el modo de argumentación de un párrafo y de un texto.

- Escribir una introducción general para presentar el tema.

- Manifestar claramente la tesis principal.

- Desarrollar adecuadamente el cuerpo argumentativo a través de la ejemplificación, la adición de argumentos y el razonamiento deductivo.

- Proponer una conclusión.

\subsection{Contenidos de la unidad didáctica}

\subsubsection{Conceptos}

1. El texto argumentativo:

1.1. Características.

1.2. Modalidades.

1.3. Estructura. 
2. Cohesión y coherencia textual:

2.1. Unidades lógico-formales: texto, párrafo y oración.

2.2. Elementos de cohesión formal: repeticiones, sustituciones, vínculos lógicosintácticos (nexos y conectivos, relaciones lógicas esenciales).

2.3. Elementos de cohesión semántica.

\subsubsection{Procedimientos}

1. Elaboración del esquema previo del texto.

2. Búsqueda y síntesis de la información necesaria.

3. Selección de datos relevantes.

4. Decisión sobre posición del enunciador y su relación con el destinatario.

5. Determinación de la tesis principal.

6. Planificación del desarrollo del cuerpo argumentativo.

7. Extracción de la conclusión adecuada.

8. Utilización de los instrumentos de autocontrol: pautas, carta de estudios, base de orientación, cuatro sinóptico de nexos.

9. Revisión y corrección de errores (faltas de ortografía, puntuación inadecuada, repeticiones léxicas, redundancias, ambigüedades, anacolutos...).

10. Análisis de las producciones propias y ajenas.

11. Imitación de modelos argumentativos.

\subsubsection{Actitudes}

1. Reconocimiento de la utilidad de planificar el texto.

2. Preocupación por la claridad argumentativa y el orden.

3. Respeto y valoración crítica por las producciones propias y ajenas.

4. Interés por la presentación puntual en la entrega de las composiciones.

5. Valoración por la participación en el trabajo en equipo.

6. Defensa de las opiniones propias y respeto por las ajenas.

\subsection{Desarrollo de las fases: descripción y temporalización de las actividades}

Las actividades de enseñanza-aprendizaje se han diseñado siguiendo, a grandes rasgos, las cuatro etapas señaladas, que se resumen en estos verbos: actuar, reflexionar, analizar y generalizar. Paso a exponer a continuación la descripción y la temporalización de las actividades realizadas durante las ocho jornadas:

\subsubsection{Fase de actuación: 1. 므 y 2. $\stackrel{a}{\text { jornadas }}$}

Los objetivos de esta fase se centran en las necesidades de comunicación de los alumnos, concretamente en la adquisición de la competencia comunicativa concretada en actos de habla. En estas jornadas se fomentan las situaciones interactivas (juego de papeles, actividades por parejas, dinámica de grupos...) y se trabaja la lengua en el aula tal como se usa habitualmente. 
En primer lugar se negoció con los alumnos el contenido y objetivos de la unidad didáctica y se les dejó elegir el contexto de su aplicación. Después de la presentación de las diferentes propuestas, se pasó a la votación de las mismas; resultó elegida la que planteaba la discusión sobre determinados temas, organizada a modo de juicio. Este juicio constó de las siguientes partes:
a) Presentación del tema a cargo de fiscales y defensores.
b) Debate entre ambos grupos.
c) Intervención del público.
d) Conclusiones de fiscales y defensores.
e) Veredicto del tribunal popular.
f) Sentencia del juez.
g) Crónica del juicio a cargo de los periodistas.

En cada fase se elaboraron escritos argumentativos que fueron leídos en clase. Para ayudarnos en esta terea, visionamos varios fragmentos de películas en las que se desarrollaban interesantes juicios -esto les motivó realmente- y las comentamos a posteriori.

El planteamiento inicial se desarrolló de la siguiente forma: primero se eligió el tema objeto de debate. Para ello cada alumno escribió un texto opinando acerca de un tema de su elección. Los textos se leyeron y comentaron en clase. Se eligió el tema que más motivó a la mayoría, "A favor o en contra de la eutanasia" (los restantes más solicitados fueron "El consumo de drogas blandas" y "La enseñanza obligatoria hasta los 16 años". A continuación se hizo una primera aproximación al tema, de modo que cada alumno expresara individualmente y por escrito lo que pensaba acerca del mismo. Después de que todos leyeran en voz alta sus textos, se establecieron los grupos. De los que se manifestaron a favor, surgió el grupo de los defensores; de los que se manifestaron en contra, el de los fiscales. De los que valoraron tanto pros como contras, sin acabar de definir su posición, se eligió el tribunal popular; y de entre éstos, el juez. El resto se convirtió en público. Una vez formados los grupos, los equipos de fiscales y defensores redactaron un texto con vistas a ser expuesto en la presentación de la causa.

Se celebraron las dos primeras partes del juicio con intervenciones orales de la mayoría de los componentes de ambos grupos -fiscales y defensores-. Mientras tanto, el resto de alumnos actuó según la función que tenía encomendada: el juez presidió la sesión, concediendo los correspondientes turnos a la defensa y a la fiscalía; el tribunal popular anotó las alegaciones de defensores y fiscales para determinar y justificar su veredicto; el público observó y anotó todo cuanto se desarrollaba en el juicio, preparando su intervención antes de las conclusiones.

Organicé y guié el desarrollo del proceso y grabé con una cámara de vídeo todo cuanto ocurría. Además corregí los textos mientras se estaban escribiendo y una vez terminados, se volvieron a revisar. Comprobé que cada texto tuviera esquema, borrador y versión final.

\subsubsection{Fase de reflexión: $3 . \stackrel{a}{\text { y }}$ 4. $\cdot^{\underline{a}}$ jornadas}

El punto de partida de las actividades de esta fase se basa en las necesidades académicas de los alumnos, es decir, aprovechar el potencial creativo de la composición 
escrita como instrumento de aprendizajes de las materias escolares. Lo que más interesa es lo que el texto dice, su contenido, si las ideas son claras, originales, creativas, si están bien ordenadas y estructuradas con argumentos sólidos. Las fuentes de escritura son básicamente bibliográficas: libros de texto, apuntes, artículos, charlas, conferencias...

Las actividades realizadas fueron las siguientes:

- Consulta en la biblioteca de distintas fuentes bibliográficas, básicamente libros de texto y diccionarios, preparadas por mí, con el fin de encontrar información sobre el texto argumentativo.

- Aplicación de las estrategias de comprensión, resumen y esquematización de textos, aprendidas en el curso anterior, cuando se trabajó el texto expositivo.

- Selección de la información relevante por grupos de 4 alumnos.

- Redacción con el profesor de un texto común para todos a partir de un esquema previo suministrado por mí y de los datos relevantes extraídos de la consulta bibliográfica, contrastados con nuevas informaciones por mí aportadas.

- Confección entre todos de un "Esquema de la argumentación" que reúne los conceptos básicos sobre la misma y que sirve como instrumento de ayuda para la redacción de textos argumentativos.

- Confección de una guión procedimental con las consignas básicas que deben tenerse en cuenta en el proceso de redacción de un texto argumentativo.

- Elaboración de una guía de consulta sobre el valor y uso de los nexos y conectivos lógicos. Ejemplificación del uso de los nexos más comunes y necesarios para argumentar.

En este apartado se trabajó el texto expositivo, que los alumnos ya habían estudiado en cursos anteriores. Resultó muy enriquecedor comprobar que la información que ellos habían encontrado se correspondía básicamente con la que yo había elaborado. De esta investigación sobre el texto argumentativo surgieron los tres últimos documentos citados. A lo largo de estas jornadas, verifiqué el grado de competencia alcanzado en la comprensión y síntesis de información, de acuerdo con la enseñado anteriormente. Proporcioné el esquema para la redacción del texto sobre la argumentación: definición, características y modalidades, estructura y elementos de cohesión lógico-sintácticos.

El método inductivo que guía esta fase permitió comprobar que los alumnos captan mejor una explicación y comprenden más a fondo la esencia de un contenido cuando éste ha sido introducido después de una serie de prácticas que lo han situado en ese contexto conceptual.

\subsubsection{Fase de análisis: $5 .^{\underline{a}}$ y $6 .^{\underline{a}}$ jornadas}

Esta tercera fase se inspiró en los contenidos gramaticales de la propia programación, aunque quedó restringida al estudio de los nexos y conectivos relacionados con aspectos como la cohesión, coherencia interna y externa y estructura del texto. Estas actividades más específicas de teoría gramatical quedan, pues, incardinadas en un proyecto de creación textual. 
Las actividades propuestas fueron las siguientes:

- Análisis de los textos escritos hasta el momento, teniendo en cuenta lo aprendido en la fase de reflexión y contando con los anteriores documentos tomados como pautas, así como con su propia creación.

- Análisis de la cinta de vídeo con las dos primeras partes del juicio.

- Análisis de textos literarios y periodísticos de otros autores (eso fue un verdadero "taller de indagación". Se llevaron a clase periódicos, libros, revistas... y, entre todos, se eligieron los materiales pertinentes.

En esta fase se aplicaron los conceptos aprendidos en la fase anterior. Se comprobó si los textos escritos y las intervenciones orales de los alumnos respondían a las características específicas de un texto argumentativo. Se analizaron textos de otros autores, verificando la riqueza y variedad de la argumentación. Se comentó el uso de los nexos y conectivos. Es básico el papel del profesor que dialoga, pregunta y comenta, actuando como estímulo y eje de las reflexiones que suscita el análisis textual.

\subsubsection{Fase de generalización: 7.ㄹ y 8..$^{a}$ jornadas}

La clase de lengua es el lugar del aprendizaje de una función comunicativa determinada, por eso se busca la interactividad en el aula.

Las actividades que se trabajaron en esta fase fueron:

- Preparación y celebración de la tercera parte del juicio, con la intervención del público valorando el desarrollo del mismo con las conclusiones de fiscales y defensores, con el veredicto del tribunal popular y con la sentencia del juez.

- Redacción de textos argumentativos a partir de temas sugeridos por los propios alumnos.

- Confección de un dossier final que recopila todos los textos redactados y analizados a lo largo de la unidad didáctica.

- Antología de los textos más significativos de la unidad. Esta antología se compuso informáticamente -con un procesador de textos y en el aula de informática- y se distribuyó a todos los componentes del grupo.

En esta fase se buscaba consolidar lo aprendido aplicándolo a los nuevos escritos. Se grabó con el casete la preparación de las conclusiones para comprobar cómo se habían asimilado los conceptos y procedimientos trabajados en las dos fases anteriores. La sesión del juicio se volvió a grabar en vídeo. Todos los grupos aportaron sus respectivos textos que presentaban una notable mejoría en comparación con los de los primeros días. Algunas de las tareas, como escribir textos, se realizaron en casa. Finalmente se procedió a la edición de un documento que mostraba el trabajo realizado a través de la unidad.

Aclaré los objetivos, sinteticé lo aprendido y guié a los alumnos, a través del estímulo, en la consecución de composiciones escritas bien acabadas. Facilité la reflexión por medio de la comparación y el contraste entre los textos del inicio de la unidad y los textos finales. Corregí e intervine activamente, junto con los alumnos, en la selección de los textos que formaron la antología y acordé con los alumnos la fecha límite para la edición y distribución de la misma. 


\subsection{Orientaciones didácticas generales y metodología}

En cuanto a las bases psicopedagógicas en que se apoya la unidad, se sigue, de modo general, el aprendizaje constructivo y significativo, esto es, es el propio alumno quien, de una manera activa, extraerá desde sus esquemas cognitivos ya elaborados los nuevos conceptos que se quieren fijar. El profesor, más que instructor, es orientador, colaborador y planificador. La metodología y didáctica seguida se puede concretar en los siguientes aspectos:

1. La metodología es prioritariamente activa, propiciando el trabajo personal, tanto de forma individual como colectiva.

2. Todo el proceso de la unidad está concebido como un desarrollo a lo largo de las jornadas establecidas.

3. La unidad no se plantea como algo cerrado.

4. Las actividades que se plantean tienen, por consiguiente, un carácter orientador, susceptible de variaciones.

5. Se tiene en cuenta la atención a la diversidad y el establecimiento de estrategias de recuperación, en caso de necesidad.

\subsection{Evaluación de la unidad didáctica}

\subsubsection{Evaluación de los alumnos}

El diagnóstico inicial llevado a cabo a través de los primeros textos de los alumnos mostró que la mayoría de ellos tenían considerables lagunas en la estructuración lógica del texto; lagunas debidas al desconocimiento de la relación jerárquica entre las ideas (pasar de lo particular a lo general y viceversa; organizar adecuadamente los argumentos de apoyo a la tesis principal o determinar con claridad esta misma; relacionar causa-consecuencia; fijar las conclusiones...), a la inadecuada utilización, en algunos casos, de los nexos y a la distribución incoherente del contenido en párrafos, a menudo, mal secuenciados desde el punto de vista de la lógica del texto. Este conocimiento previo del nivel determinó el posterior desarrollo de la secuencia, que concedió mayor importancia a aquellos aspectos que los alumnos menos dominaban.

La mayoría de los instrumentos diseñados se encaminaron a fomentar un autocontrol sobre el proceso de redacción de los textos y sobre el proceso de aprendizaje de los contenidos específicos relativos a la argumentación. Se convirtieron, por tanto, en eficaces aliados de la evaluación formativa. La "Pauta de evaluación de la unidad" (se incluye a continuación) con su cuadro de especificaciones para la corrección de los ejercicios escritos, la observación en clase del trabajo individual y de los grupos, así como las grabaciones de audio y vídeo y el dossier recopilatorio fueron los puntos de referencia que determinaron la nota final de cada alumno. 
PAUTA DE EVALUACIÓN DE LA UNIDAD

"Escribir textos argumentativos"

Alumno

Curso

Tema

\section{OBJETIVOS}

\section{Estimación y observaciones}

\section{ORGANIZACIÓN DEL TEXTO:}

Establecer un plan o esquema

Elegir y dar coherencia al enunciador

Tener en cuenta el destinatario

Escribir un borrador previo

Coregir el texto

Entregar una versión definitiva

\section{CONTENIDO DEL TEXTO:}

Escribir una introducción

Manifestar claramente la tesis principal

Desarrollar adecuadamente el cuerpo argumentativo

Utilizar con precisión nexos y conectivos lógicos

Proponer una conclusión

Usar un léxico adecuado

\section{UTILIZACIÓN DEL CÓDIGO ESCRITO:}

Construir oraciones bien estructuradas

Utilizar las normas de puntuación

Respetar las reglas de tiempo y persona verbales

Respetar las reglas ortográficas

Escribir con letra legible

\section{Estimación para cada objetivo:}

3, BIEN; 2, SUFICIENTE; 1, INSUFICIENTE; O, DEFICIENTE. 
Esta pauta de evaluación se apoya, asimismo, en las siguientes técnicas ya explicadas anteriormente:

1. La observación individualizada del alumno.

2. El cuaderno personal.

3. Autoevaluación razonada.

4. Texto argumentativo final.

5. Antología de los textos producidos.

\subsubsection{Evaluación de la unidad}

No hay que olvidar que no sólo el alumno es objeto de evaluación, sino también la unidad. Si ésta se adecua a los objetivos generales de la etapa y del área, su desarrollo, los materiales utilizados y, en último término, si entra en las necesidades e intereses del propio alumno. Es decir, se evalúa el proceso y el resultado.

Según las valoraciones efectuadas por los propios alumnos, la secuencia les resultó muy estimulante; lo más motivador y gratificante fueron los trabajos en grupo (preparando y escribiendo textos) y la celebración del juicio en todas sus partes. La mayoría reconocieron haber aprendido a argumentar, a saber disponer adecuadamente los elementos que constituyen un texto argumentativo y a utilizar la modalidad más idónea para sus propósitos comunicativos. Consideraron muy útiles las técnicas practicadas en clase de preparación previa de las redacciones (torbellino de ideas, discusiones, esquemas...) y la frecuente lectura de los textos y su valoración crítica. Por último, reconocieron haberse acercado a la reflexión gramatical con mayor interés y predisposición que en anteriores ocasiones.

Por mi parte, considero que las actividades, al estimular al mismo tiempo actuación y recepción reflexiva, facilitaron el trabajo en equipo y la implicación del alumnado en el aprendizaje, sobre todo en los aspectos de autocontrol y autoevaluación de las tareas. Hay que destacar el interés tomado por la redacción de este tipo de textos, hasta el punto de superar los límites establecidos en el diseño de la unidad, ya que los propios textos de los compañeros se convirtieron en objeto de polémica e incitaron a una respuesta escrita, sin que se hubiera solicitado.

Los contenidos más teóricos fueron perfectamente asumidos por la mayoría, sobre todo los relativos a la estructura y a las modalidades de la argumentación y al uso y funciones de los nexos, aunque, quizá, habría que insistir más en la distinción y relación entre coherencia semántica y cohesión textual.

\section{Referencias bibliográficas}

BASART, D.G. (1995): "Elementos para una didáctica de la argumentación en la escuela primaria", en Comunicación, Lenguaje y Educación, 25, págs. 41-50.

CAMPS, A. (1995): "Aprender a escribir textos argumentativos: características dialógicas de la argumentación escrita", en Comunicación, Lenguaje y Educación, 25, pág. 51-63. 
CAMPS, A. y DOLZ, J (1995): "Introducción. Enseñar a argumentar: un desafío para la escuela actual", en Comunicación, Lenguaje y Educación, 25, págs. 5-8.

CASTELLÓ, M. y MONEREO, C. (1996): “Un estudio empírico sobre la enseñanza y el aprendizaje de estrategias para la composición escrita de textos argumentativos", en Infancia y aprendizaje, 74, págs. 39-55.

COTTERON, J. (1995): "¿Secuencias didácticas para enseñar a argumentar en la escuela primaria?", en Comunicación, Lenguaje y Educación, 25, págs. 79-94.

DOLZ, J. (1993): “La argumentación", en Cuadernos de Pedagogía, Monográfico Leer y escribir, 216, págs. 68-70.

- (1994): "La interacción de las actividades orales y escritas en la enseñanza de la argumentación", en Comunicación, Lenguaje y Educación, 23, págs. 17-27.

-- (1995): "Escribir textos argumentativos para mejorar su comprensión", en Comunicación, Lenguaje y Educación, 25, págs. 65-77.

DOLZ, J.; PASQUIER, A. y BRONCKART, J-P. (1993): "La adquisición de los discursos: ¿Competencia emergente o aprendizaje de diversas capacidades verbales?", en Actas de las Jornadas sobre la enseñanza de las lenguas en la EOI. Castellón de la Plana.

PERELMAN, CH. y OLBRECHT-TYTECHA, L. (1989): Tratado de argumentación (La nueva retórica). Gredos, Madrid. 